\title{
BIBLIOGRAPHY
}

1. E. Artin, The gamma function, Holt, New York, 1964.

2. Philip J. Davis and Philip Rabinowitz, Numerical integration, Blaisdell, Waltham, Mass., 1967.

3. L. Hörmander, On the division of distribution by polynomials, Ark. Mat. 3 (1958), 555-568.

4. K. Knopp, Theory and application of infinite series, Blachie, London, 1951.

5. K. Mahler, Über einer Satz von Mellin, Math. Ann. 100 (1928), 384-395.

6. T. Ono, An integral attached to a hypersurface, Amer. Math. J. (to appear).

University of Pennsylvania, Philadelphia, Pennsyluania 19104

\section{FUNCTIONS OF BOUNDED CONVEXITY}

BY A. WAYNE ROBERTS AND DALE E. VARBERG ${ }^{1}$

Communicated by R. Creighton Buck, October 31, 1968

1. Introduction. Functions of bounded variation on $[a, b]$ are those functions for which

$$
V_{a}^{b}(f)=\operatorname{Sup}_{\boldsymbol{P}} V(f, P)=\operatorname{Sup}_{\boldsymbol{P}} \sum_{j=1}^{n}\left|\Delta f_{j}\right|
$$

is finite. An important theorem about the set $B V[a, b]$ of all such functions says that this set may be characterized as the set of all functions representable as the difference of two nondecreasing functions. Stated with less precision but more suggestion for our purposes, $B V[a, b]$ is the set of all functions representable as the difference of two functions with nonnegative first derivatives. It is then natural to consider the set of all functions representable as the difference of two functions with nonnegative second derivatives (convex functions, roughly speaking).

We begin our study with an expression that plays the role of (1). For a partition $P=\left\{a=x_{1}<x_{2}<\cdots<x_{n}=b\right\}$, let $\square f_{j}$ $=\left[f\left(x_{j}\right)-f\left(x_{j-1}\right) /\left(x_{j}-x_{j-1}\right)\right]$.

Definition 1. For $f:[a, b] \rightarrow R$, let

$$
K_{a}^{b}(f)=\operatorname{Sup}_{\boldsymbol{P}} K(f, P)=\operatorname{Sup}_{\boldsymbol{P}} \sum_{j=1}^{n-1}\left|\square f_{j+1}-\square f_{j}\right| \text {. }
$$

1 The second author was supported by the National Science Foundation under grant number GP-7843. 
If $K_{a}^{b}(f)<\infty$, we say $f$ is of bounded convexity on $[a, b](f \in B C[a, b])$ and we call $K_{a}^{b}(f)$ the total convexity of $f$ on $[a, b]$.

This definition gives rise to a host of interesting consequences which we outline below. Full proofs of the indicated results will appear elsewhere.

\section{The basic theory.}

THEOREM 1. If $K_{a}^{b}(f)<\infty$, then $f_{+}^{\prime}(x)$ exists on $[a, b)$ and $f_{-}^{\prime}(x)$ exists on $(a, b]$. Moreover, both one-sided derivatives are bounded on the domains where they exist.

From Theorem 1 it follows that functions of bounded convexity are continuous, have finite derivatives except for at most countably many points and are absolutely continuous [6, Theorem 4]. And once guaranteed the existence of left and right derivatives, we may prove

THEOREM 2. Let $a<c<b$. Then $K_{a}^{o}(f)<\infty$ if and only if $K_{a}^{c}(f)$ and $K_{c}^{b}(f)$ are finite, and in this case

$$
K_{a}^{b}(f)=K_{a}^{0}(f)+K_{c}^{b}(f)+\left|f_{+}^{\prime}(c)-f_{-}^{\prime}(c)\right| .
$$

For a function $f$ convex on $[a, b]$, (2) becomes $f_{+}^{\prime}(a)-f_{-}^{\prime}(b)$, so $K_{a}^{b}(f)<\infty$ if and only if both $f_{+}^{\prime}(a)$ and $f_{-}^{\prime}(b)$ are finite. For this reason, we cannot expect to identify $B C[a, b]$ with the collection of functions representable as the difference of any two convex functions. But by restricting attention to convex functions of bounded convexity, we can obtain the anticipated characterization of $B C[a, b]$. Toward this end, for $f \in B C[a, b]$, we define the following functions.

$$
\begin{array}{rlrl}
t(x) & =K_{a}^{*}(f) \quad \text { with } t(a)=0 . & \\
f^{*}(x) & =f_{-}^{\prime}(x) \quad \text { for } x \in(a, b] & \text { and } \quad f^{*}(a)=f_{+}^{\prime}(a) . \\
p(x) & =\frac{1}{2}\left[t(x)+f^{*}(x)-f^{*}(a)\right] ; & & P(x)=\int_{a}^{x} p(u) d u . \\
n(x) & =\frac{1}{2}\left[t(x)-f^{*}(x)+f^{*}(a)\right] ; & N(x)=\int_{a}^{x} n(u) d u .
\end{array}
$$

The key is to demonstrate that both $p$ and $n$ are nondecreasing functions, since it then follows that $P$ and $N$ are convex. The desired representation comes from the fact that the (absolutely continuous) function $f$ may be written as 


$$
\begin{aligned}
f(x)-f(a)-f_{+}^{\prime}(a)(x-a) & =\int_{a}^{x}\left[f^{\prime}(u)-f_{+}^{\prime}(a)\right] d u \\
& =\int_{a}^{x}[p(u)-n(u)] d u=P(x)-N(x) .
\end{aligned}
$$

Theorem 3. If $K_{a}^{b}(f)<\infty$, then

$$
f(x)=P(x)-N(x)+\left[f_{+}^{\prime}(a)(x-a)+f(a)\right]
$$

and $K_{a}^{b}(f)=K_{a}^{b}(P)+K_{a}^{b}(N)=V_{a}^{b}\left(f^{*}\right)$, where $V_{a}^{b}\left(f^{*}\right)$ is the total variation of $f^{*}$ on $[a, b]$.

The bracketed term, being linear, can be combined with either $P$ or $N$ to give a representation of $f$ as the difference of two convex functions in $B C[a, b]$. The surprising relation to $V_{a}^{b}\left(f^{*}\right)$ gives several interesting corollaries, of which we here include just one.

CoRollary. If $f$ is twice differentiable with $f^{\prime \prime}$ integrable on $[a, b]$, then $K_{a}^{b}(f)<\infty$ and

$$
K_{a}^{b}(f)=\int_{a}^{b}\left|f^{\prime \prime}(u)\right| d u .
$$

3. The space $B C[a, b]$ and related spaces. It is easy to see that $B C[a, b]$ is a normed linear space with norm

$$
\|f\|=K_{a}^{b}(f)+\left|f_{+}^{\prime}(a)\right|+|f(a)| .
$$

The subspace consisting of those functions $f$ for which $f_{+}^{\prime}(a)=f(a)=0$ will be denoted by $B C N[a, b]$. Two related spaces are of interest. The functions of bounded variation, $B V[a, b]$, normed by $\||g|\|$ $=V_{a}^{b}(g)+|g(a)|$ are known [5, p. 103] to form a Banach Space. The subspace of functions $g$ which are left continuous on $(a, b]$, right continuous at $a$, and which satisfy $g(a)=0$ will be denoted by $B V N[a, b]$. A fact crucial to our development here is that $B V N[a, b]$ with the norm $\|\mid\|$ is also a Banach Space. After proving this, we are able to prove, using $f^{*}$ as defined above,

THEOREM 4. $B C N[a, b]$ is congruent (isometrically isomorphic) to $B V N[a, b]$ under the correspondence $f \leftrightarrow f^{*}$. Therefore, $B C N[a, b]$ is a Banach Space.

As to the space $B C[a, b]$ itself, we need to introduce the space $R \times R \times B V N[a, b]$ normed by $\|(\alpha, \beta, g)\|=|\alpha|+|\beta|+V_{a}^{b}(g)$. This 
product of Banach Spaces is again a Banach Space, so Theorem 5 settles the question of completeness for $B C[a, b]$.

TheOREM 5. $B C[a, b]$ is congruent to $R \times R \times B V N[a, b]$.

The space $B V N[a, b]$ is congruent to $N B V(a, b)$ of Dunford and Schwartz [1, pp. 241, 342-344, 378]. Hence, Theorem 5 enables us to answer for $B C[a, b]$ many of the questions usually asked about Banach Spaces.

4. Vector valued functions. For a vector valued function, the obvious generalization of $(2)$ is

$$
K_{a}^{b}(f)=\operatorname{Sup}_{P} \sum_{j=1}^{n-1}\left\|\square f_{j+1}-\square f_{j}\right\| .
$$

If $\boldsymbol{f}(t)=\left(f_{1}(t), \cdots, f_{m}(t)\right)$, then the inequality

$$
\left|v_{i}\right| \leqq\left\|\left(v_{1}, v_{2}, \cdots, v_{m}\right)\right\| \leqq\left|v_{1}\right|+\cdots+\left|v_{m}\right|
$$

can be used to establish the not surprising

Theorem 6. $K_{a}^{b}(f)<\infty$ if and only if $K_{a}^{b}\left(f_{i}\right)<\infty$ for $i=1, \cdots, m$.

The concepts of differentiability, bounded variation, and absolute continuity for $f$ are closely related to these concepts for the coordinate functions [4, p. 382ff], so Theorem 6 has all the expected straightforward consequences of $\S 2$. One of these results relates our work to that of classical differential geometry. The analog of the corollary to Theorem 3 is

CoROllary. If $\boldsymbol{f}$ is twice differentiable with $\boldsymbol{f}^{\prime \prime}$ integrable on $[a, b]$, then

$$
K_{a}^{b}(\boldsymbol{f})=\int_{a}^{b}\left\|\boldsymbol{f}^{\prime \prime}(t)\right\| d t .
$$

Now in the case where $f$ is the parameterization of a curve $C$ with respect to arc length $s,\left\|f^{\prime \prime}(s)\right\|$ is equal to the curvature $\kappa(s)$ of the curve, and (3) is what is known in the literature [2, p. 252], [3, p. 204] as the total or integral curvature of $C$.

It should be emphasized that for a given curve $C$, (3) will be the total curvature of $C$ in general only if $f$ is the representation of $C$ with respect to arc length. We are able to show by example that for different representations of the same curve, one may obtain any positive value including $+\infty$ from (3). However, there is a formula for obtaining the total curvature of a curve $C$ directly from an arbitrary representation $f$. 
Many of the ideas which we have discussed also have generalizations for functions $f: R^{m} \rightarrow R$. We shall not take space to discuss them here.

\section{REFERENCES}

1. N. Dunford and J. T. Schwartz, Linear operators, Part 1, Interscience, New York, 1958.

2. H. W. Guggenheimer, Differential geometry, McGraw-Hill, New York, 1963.

3. D. J. Struik, Differential geometry, 2nd ed., Addison-Wesley, Reading, Mass., 1961.

4. A. E. Taylor, General theory of functions and integration, Blaisdell, New York, 1965.

5. - Introduction to functional analysis, Wiley, New York, 1967.

6. D. E. Varberg, On absolutely continuous functions, Amer. Math. Monthly 72 (1965), 831-841.

Macalester College, St. Paul, Minnesota 55101 and

Hamline University, St. Paul, Minnesota 55101 